This is a self-archived version of an original article. This version may differ from the original in pagination and typographic details.

Author(s): Zhu, Yongjie; Li, Xueqiao; Ristaniemi, Tapani; Cong, Fengyu

Title: Measuring the Task Induced Oscillatory Brain Activity Using Tensor Decomposition

Year: 2019

Version: Accepted version (Final draft)

Copyright: @ 2019 IEEE

Rights: In Copyright

Rights url: http://rightsstatements.org/page//nC/1.0/?language=en

Please cite the original version:

Zhu, Y., Li, X., Ristaniemi, T., \& Cong, F. (2019). Measuring the Task Induced Oscillatory Brain Activity Using Tensor Decomposition. In ICASSP 2019 : Proceedings of the 2019 IEEE International Conference on Acoustics, Speech and Signal Processing (pp. 8593-8597). IEEE. Proceedings of the IEEE International Conference on Acoustics, Speech, and Signal Processing. https://doi.org/10.1109/ICASSP.2019.8682355 


\title{
MEASURING THE TASK INDUCED OSCILLATORY BRAIN ACTIVITY USING TENSOR DECOMPOSITION \\ Yongjie Zhu ${ }^{1,2}$, Xueqiao $\mathrm{Li}^{3}$, Tapani Ristaniemi ${ }^{2}$, Fengyu Cong ${ }^{1,2}$
}

\author{
${ }^{1}$ School of Biomedical Engineering, Faculty of Electronic and Electrical Engineering, Dalian University \\ of Technology, 116024, Dalian, China \\ ${ }^{2}$ Faculty of Information Technology, University of Jyvaskyla, Jyväskylä,40014, Finland \\ ${ }^{3}$ Department of Psychology, University of Jyvaskyla, Jyväskylä, 40014, Finland
}

\begin{abstract}
The characterization of dynamic electrophysiological brain activity, which form and dissolve in order to support ongoing cognitive function, is one of the most important goals in neuroscience. Here, we introduce a method with tensor decomposition for measuring the task-induced oscillations in the human brain using electroencephalography (EEG). The time frequency representation of source-reconstructed singletrail EEG data constructed a third-order tensor with three factors of time $*$ trails, frequency and source points. We then used a non-negative Canonical Polyadic decomposition (NCPD) to identify the temporal, spectral and spatial changes in electrophysiological brain activity. We validate this method using both simulation EEG data and real EEG data recorded during a task of irony comprehension. The results demonstrated that proposed method can track dynamics of the temporal-spectral modes of the rhythm in the brain on a timescale commensurate to the task they are undertaking.
\end{abstract}

Index Terms - EEG, source localization, neural oscillations, tensor decomposition.

\section{INTRODUCTION}

During the past decade, the characterization of brain functional networks and their dynamics has become an important field of study [1]. Most efforts focusing on the functional networks have been made through functional magnetic resonance imaging (fMRI) technique due to high spatial resolution [2]. Unfortunately, fMRI temporal resolution is limited since it indirectly measures the consequences of neural activity. The electrophysiological underpinnings of the human brain are not yet fully understood through fMRI. The direct non-invasive measures of neural activity such as electro- or magnetoencephalography (EEG/MEG) provide a means to study the neural oscillations.

The EEG consists of the activity of an ensemble of generators producing rhythmic activity in several frequency ranges [3]. By application of sensory stimulation these generators are coupled and act together in a coherent way. This synchronization and enhancement of EEG activity gives rise to 'evoked' or 'induced' oscillations (the former being phase-locked to the event, the latter not) $[3,4]$. To obtain the data representation of the evoked oscillations, the single trial EEG data were first averaged and then transformed to the time-frequency domain by means of wavelet analysis. The obtained data representations were with multi modes since EEG had many channels in sensor space. The analysis for multi-way data (channel $\times$ time $\times$ frequency) of the evoked oscillation based on tensor decomposition has been studied [5-7] (for review see [8]). In contrast, the data of induced oscillation can be generated by transforming the single trial EEG to time-frequency domain, which resulted in another data formation with channel $\times$ time $*$ trial $\times$ frequency. In addition, to examine functional brain structure, source reconstruction techniques are applied to sensor-level EEG data, which can somehow overcome the limited spatial resolution of the EEG $[9,10]$. Thus, a new data representation can be generated with source $\times$ time $*$ trial $\times$ frequency. CANDECOMP/PARAFAC (CP), as a basic tensor decomposition method, can be applied to sourcereconstructed data to extract task-induced neural oscillations.

In this study, we proposed a method based on NCPD for measuring the task-induced oscillations in the human brain. The time frequency representation of single-trail sourcereconstructed EEG data constructed a third-order tensor with three factors of time $*$ trails, frequency and source space. NCPD was performed to identify the temporal, spectral and spatial changes in electrophysiological brain activity. The proposed method was validated using both simulated EEG data and real EEG data recorded during a task of irony comprehension. The results demonstrated that proposed method can tracks dynamics of the temporal-spectral modes of the rhythm in the brain.

\section{MATERIALS AND METHODS}

\subsection{Data description}

\subsubsection{Simulated data}

We simulated EEG data using Brainstorm toolbox [11]. Three oscillating current dipoles perpendicular to the cortical surface were placed pre-selected brain regions (Fig.1). The duration of each trail of the simulated measurement was 1400 
ms from -200 to $1200 \mathrm{~ms}$. Each oscillatory source, generated using different frequent sine wave $(8 \mathrm{~Hz}, 15 \mathrm{~Hz}, 25 \mathrm{~Hz}$, see Fig.1), was amplitude modulated by a different smoothed Hanning widow (Fig. 2) with a SNR of $20 \mathrm{~dB}$. Next, we applied a forward solution with a boundary-element conductor model from template anatomy to simulate the 128channel EEG data. Finally, to make the simulations more realistic, the magnitudes of different trails were different and were normal distribution.

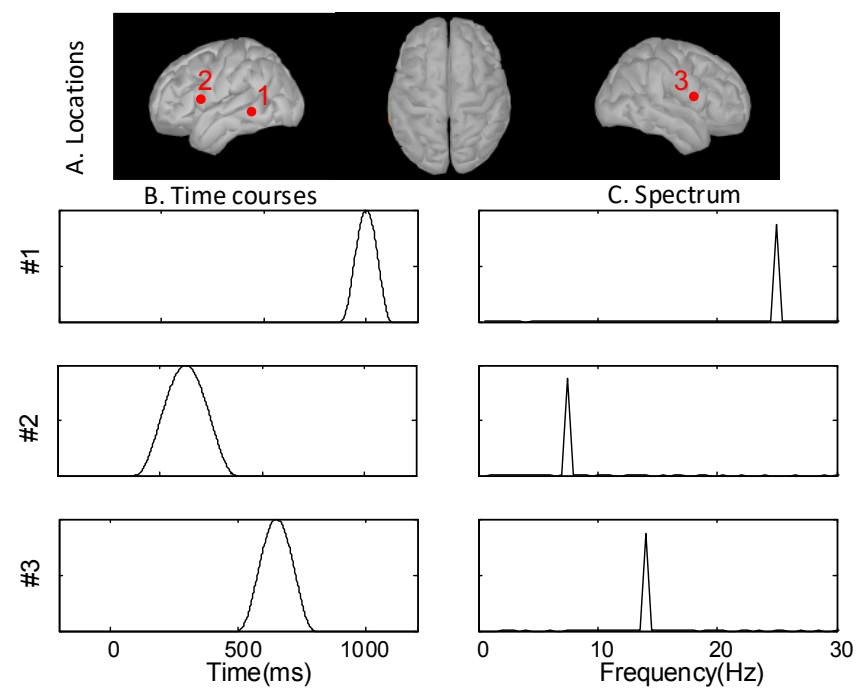

Fig. 1. Locations (A) of the simulated oscillatory current sources on an inflated and flattened brain surface and the time courses (B) and spectrum (C) of the three sources.

\subsubsection{Experimental data}

The data was collected at the University of Jyvaskyla, Finland. Thirty-eight participants were included in the final sample, in which there were 17 dysphoric participants and 21 control participants. The study was approved by the Ethics Committee of the University of Jyvaskyla. Stimuli with onesentence spoken lines and colored pictures were applied. Each stimulus trial consisted of an introductory sentence, a contextual picture, and a commenting sentence. There were two different types of stimuli (two conditions). If the keyword in commenting sentence was semantically congruent with the content of the contextual pictures, the trail provided a neutral meaning; otherwise, the keyword was semantically incongruent with the picture composed an ironic meaning. The commenting sentences were presented twice to the participants combined with the congruent and incongruent contextual pictures. There were 90 trials in each condition.

EEG data were collected by NeurOne system (Bittium Biosignals Ltd, Kuopio, Finland) with a 128-Channel Net (HydroCel Geodesic Sensor Net, Electric Geodesic Inc, USA), and preprocessed using EEGlab [12]. They were down-sampled to $250 \mathrm{~Hz}$ to reduce the size of datasets without losing important data information. The $0.5 \mathrm{~Hz}$ highpass and $20 \mathrm{~Hz}$ low-pass filters were applied on EEG data. Next, EEG data were visually checked and the bad channels were interpolated using a spherical spline model. After this,
EEG data was extracted into $1100 \mathrm{~ms}$ long segments relative to the onset of keywords, starting from $100 \mathrm{~ms}$ before the presentation of keywords. Segments whose maximum exceeds $150 \mu \mathrm{V}$ for all channels were rejected. Hereinafter, when EEG is mentioned, it means the preprocessed one.

In order to extract task-induced neural oscillations, the data used in this study were from the control group with incongruent (ironic) stimuli. It should be noted that we do not intend to examine the difference between groups or stimulus.

\subsection{Third-order tensor of source-level EEG data}

The forward model and the inverse model were computed with a MATLAB toolbox Brainstorm [11]. The forward model was calculated using the symmetric boundary element method and default MNI MRI template (Colin 27). Preprocessed single-trial data were used to compute the inverse model, which was estimated using the weighted Minimum Norm Estimate. Finally, activation time-courses at 4003 vertices were estimated.

Spectral decomposition of source-reconstructed EEG from single trials was conducted with Morlet wavelet. 275 linearly spaced time points form $-100 \mathrm{~ms}$ to $1000 \mathrm{~ms}$ and 37 frequency points linearly spaced between $2 \mathrm{~Hz}$ and $20 \mathrm{~Hz}$ were estimated for each trial. Therefore, for every subject, we obtained a $3 \mathrm{D}$ tensor of 37 (frequency points) $\times 275 *^{\text {' number }}$ of trials' (time points) $\times 4003$ (source points). Absolute values of the decomposed data were analyzed to investigate task-induced changes in oscillatory power.

\subsection{Nonnegative Canonical polyadic decomposition (CPD)}

In this paper, we denote a scalar variable by lowercase letter, such as $\mathrm{x}$; a vector by boldface lowercase letter, such as $\boldsymbol{x}$; a matrix by boldface uppercase letter, such as $\boldsymbol{X}$; and a high order tensor by boldface script letter, such as $\boldsymbol{X}$. Operator o represents outer product of vectors, $\circledast$ denotes the Hadarmard product, 【 \ represents Kruskal operator, and \|\|$_{F}$ means Frobenius norm. Nonnegative CANDECOMP/PARAFAC decomposition is abbreviated as NCP for convenience in following contents.

The NCP model [13] can be formulated as follows. For a given $N$ th-order tensor $\boldsymbol{X} \in \mathbb{R}_{+}^{\boldsymbol{I}_{1} \times \mathbf{I}_{2} \times \cdots \times \boldsymbol{I}_{N}}$ performing a factorization into a set of $N$ unknown non-negative matrices whose elements are non-negative: $\boldsymbol{U}^{(n)}=$ $\left[\boldsymbol{u}_{1}^{(n)}, \boldsymbol{u}_{2}^{(n)}, \cdots, \boldsymbol{u}_{J}^{(n)}\right] \in \mathbb{R}_{+}^{I_{n} \times J}(n=1,2, \cdots, N) \quad$ can $\quad$ be described as:

$$
x \approx \llbracket U^{(1)}, \cdots, U^{(N)} \rrbracket,
$$

where $J$ is the number of extracted components, $I_{n}$ is the size in mode- $n$, The Kruskal operator for estimated non-negative matrices in (1) can be represented by the sum of $J$ rank-1 tensors in outer productor form:

$$
\llbracket \boldsymbol{U}^{(1)}, \cdots, \boldsymbol{U}^{(N)} \rrbracket=\sum_{j=1}^{J} \boldsymbol{u}_{j}=\sum_{j=1}^{J} \boldsymbol{u}_{j}^{(1) \circ} \boldsymbol{u}_{j}^{(2) \circ} \cdots{ }^{\circ} \boldsymbol{u}_{j}^{(N)},
$$


where $\boldsymbol{U}_{j}(j=1,2, \cdots, J)$ are the rank-1 tensors. The target of NCP is to obtain the suitable $\boldsymbol{U}^{(n)}$ and one $\mathbf{J}$ here is defined to correspond to one NCP model. Each factor $\boldsymbol{U}^{(n)}$ explains the data tensor along a corresponding mode. Hence, one factor can be considered as features of the data onto the subspace spanned by the others. Most algorithms for NCP are to minimize a squared Euclidean distance as the following optimization problem:

$$
\min _{\boldsymbol{U}^{(1)}, \cdots, \boldsymbol{U}^{(N)}} \frac{1}{2}\left\|\boldsymbol{X}-\llbracket \boldsymbol{U}^{(1)}, \cdots, \boldsymbol{U}^{(N)} \rrbracket\right\|_{F}^{2} .
$$

In this paper, we applied the hierarchical alternating least squares (HALS) algorithm whose simplified version for NMF has been proved to be superior to the multiplicative algorithms [14]. The HALS is related to the column-wise version of the ALS algorithm for 3-D data [5]. The HALS algorithm used in this study sequentially updates components $\mathrm{u}$ by a simple update rule

$$
\boldsymbol{u}_{j}^{(n)} \leftarrow \boldsymbol{X} \overline{\times}_{-n}\left\{\boldsymbol{u}_{j}\right\}-\boldsymbol{U}_{-j}^{(n)}\left\{\boldsymbol{U}_{-j}^{T} \boldsymbol{u}_{j}\right\}^{\circledast-n},
$$

where $\boldsymbol{X} \bar{x}_{-n}\left\{\boldsymbol{u}_{j}\right\}$ is sequentially computed as the $(N-1)$ tensor-vector multiplications among all modes, but mode-n. It should be noted that this study does not intend to propose an NCP algorithm. Therefore, any NCP algorithm can work for the data. In this paper, the CPD was performed to the third-order data $(N=3)$. After decomposition, the $j$ th component containing spectral, temporal, spatial factor can be represented by $\boldsymbol{u}_{j}=\boldsymbol{u}_{j}^{(1)}{ }^{\circ} \boldsymbol{u}_{j}^{(2)}{ }^{\circ} \boldsymbol{u}_{j}^{(3)}$ according to Eqn (2).

\subsection{Component number estimation}

In the application of tensor decomposition to EEG data, it is necessary to determine a proper component number, which is the rank-1 tensor number $J$ in (2). Determining this number is very important to $\mathrm{NCP}$ because different numbers in different quantitative levels may probably correspond to very different decomposition results. In this study, DIFFIT (difference of fit) method was applied to determine the number of components [15]. DIFFIT measures the change of the fit (explained variance of the raw data by the proposed model) and the core tensor of the decomposition among a number of models [5]. We run ten times for each $J$ and average the fits to obtain a more precise estimation. After DIFFIT estimation, $J=5$ was selected for simulated dataset and $J=10$ for real EEG dataset.

\subsection{Testing for task-related brain activity}

After tensor decomposition, a set of $J$ brain activity, showing interactions among spectral temporal and spatial modes, were yielded. In all tensor-based methods, it is a general question that which components extracted need to be retained and which just reflect noise. In this study, the statistical significance of each obtained component was accessed by a permutation procedure based on surrogate data [16]. Phaserandomized surrogate time courses of equal mean and autocorrelation to the extracted temporal factor of the component were obtained. The phase-randomization was computed by rotating the phase $\phi(f)$ by an independent random variable $\varphi(f)$ which was uniformly chosen in the range of $[0,2 \pi)[16]$.

We first averaged the time courses matrix $\boldsymbol{U}^{(2)}$ over all trials in all subjects yielding a new matrix, $\overline{\boldsymbol{U}}^{(2)}$, containing $J$ trial averaged time courses of component. The size of $\overline{\boldsymbol{U}}^{(2)}$ was $N_{\text {trial }} \times J$, (where $N_{\text {trial }}$ denotes the number time points per trial; 350 for simulated data and 275 for real data). Then, the phase randomization permutation process was performed [17]. Following this, an empirical null distribution was constructed. A matrix $\widehat{\boldsymbol{U}}_{N U L L}^{(2)}$ was generated in the same way as $\overline{\boldsymbol{U}}^{(2)}$, but prior to averaging over trials, the phases of time courses for each trial were randomized. We reasoned that if the components extracted were not related to the cognitive tasks, this phase randomization would no effect on the magnitude of the trial averaged time courses, and therefore the magnitudes of fluctuations in $\widehat{\boldsymbol{U}}_{N U L L}^{(2)}$ and $\overline{\boldsymbol{U}}^{(2)}$ would match. However, if the components contained trialonset-locked increases or decreases in brain activity, then these would be maintained in $\overline{\boldsymbol{U}}^{(2)}$ but diminished in $\widehat{\boldsymbol{U}}_{\text {NULL }}^{(2)}$. This procedure was repeated 5000 times. A component was deemed significant if, at any one time point in the trial average, the associated column of $\overline{\boldsymbol{U}}^{(2)}$ fluctuated such that it fell outside a threshold defined by the null distribution. The threshold for significance was defined at 0.05 and it should be noted this was corrected by Bonferroni correction for multiple comparisons across $J$ components [17].

\section{RESULTS}

\subsection{Simulation results}

Fig. 2 shows the envelope time courses and the power spectra for all three correctly reconstructed brain networks and one noise artifact (we just present one artifact for demonstration). As can be seen that the location, spectra and the averaged time courses of the pre-set brain sources were reconstructed successfully. Fig. 2C shows the time course of the brain activity, represented as the corresponding trial averaged time courses of components in $\overline{\boldsymbol{U}}^{(2)}$. The grey area represents the null distribution generated by randomizing the phase of the trial time courses $\left(\widehat{\boldsymbol{U}}_{N U L L}^{(2)}\right)$. Again, the black line represents the average response across all trials in all subjects, and the grey distribution is the $95^{\text {th }}$ percentile threshold for the null distribution.

\subsection{Results from experimental EEG data}

Fig. 3 shows the results of our method applied to the real EEG data. Although 10 components were extracted, we just present two components that demonstrated significant task modulation. Clearly, the $1^{\text {st }}$ row of Fig. 3 indicates that the Delta brain oscillation appeared in the right temporal- 
occipital junction during $800 \mathrm{~ms}$ after onset. The $2^{\text {nd }}$ row of Fig. 3 shows that the Theta rhythm emerges in the left frontal area corresponding to Broca's area, which is significantly related with language cognition, during $400 \mathrm{~ms}$ after onset.
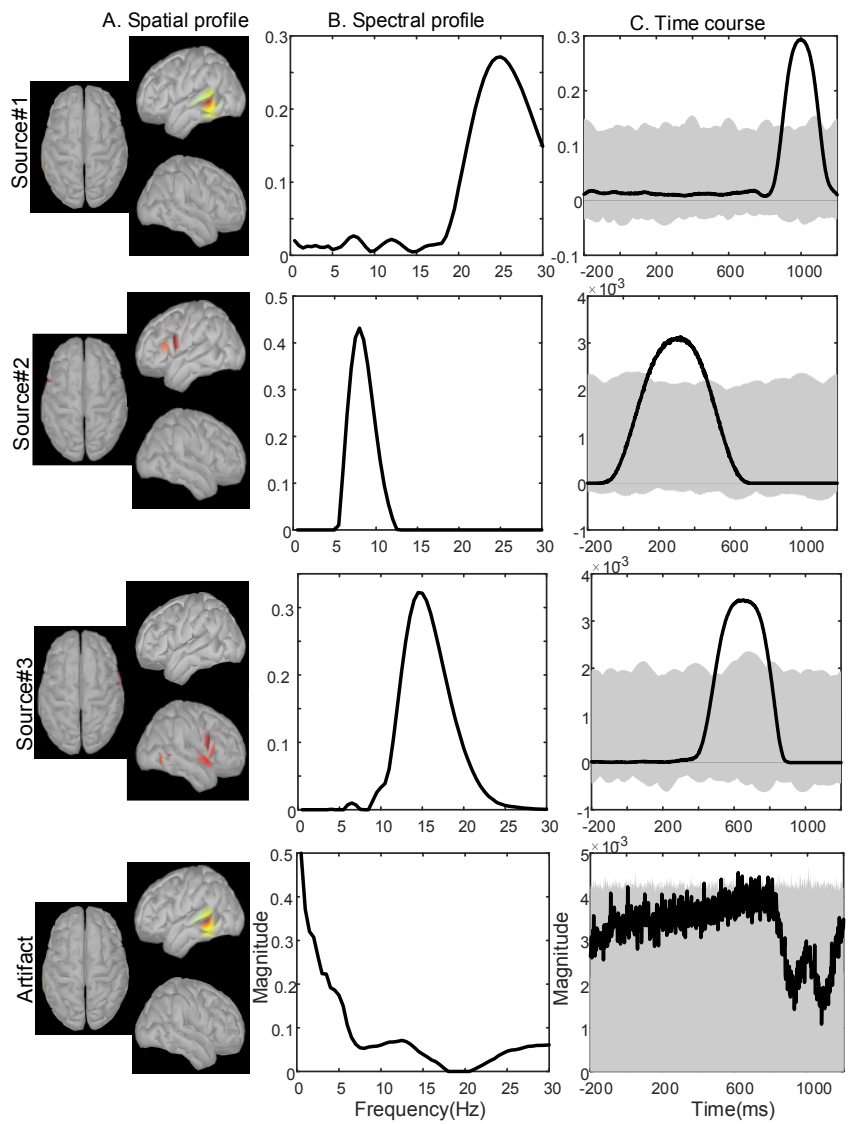

Fig. 2. Results of simulation. A: spatial maps of extracted components. B: Spectral factor of the components. C: Time courses of components, averaged across trials (black line). The grey shaded region represents the null distribution based on a hypothesis that the response is not time locked to the tasks. Significance $\left(p_{\text {corrected }}<.05\right)$ is attributed if the black line appears outside the null distribution. Rows 1 to 3 show the three induced oscillatory sources. Row 4 demonstrates an artifact.

\section{DISCUSSION}

This paper has introduced a method mainly based on tensor decomposition to extract task-induced brain oscillations, which allows characterization of transiently forming and dissolving electrophysiological brain activity. The proposed method was validated by both simulated data and real EEG data. When application to real EEG data collected from task of irony comprehension, we found brain activity of interest, which was associated with irony comprehension. The results demonstrated that the Delta rhythm was elicited in Broca's area after $400 \mathrm{~ms}$ of the ironic stimulus and the theta oscillation involved in comprehension of irony in right temporal-occipital junction after $800 \mathrm{~ms}$ of the stimulus.
Actually, such elicited brain activity in those brain regions can be expected since the previous studies has also reported that the same brain areas were associated with humor comprehension $[18,19]$.

The proposed approach is different from the previous reports where the tensor decomposition was applied to extract multi-domain feature of ERP (event-related potential) [5-7]. Here, we performed tensor decomposition to trials concatenated source-level data to extract the task-induced brain oscillation, which allows examination of temporal spectral dynamics in brain cortex during task performance or cognitive process.
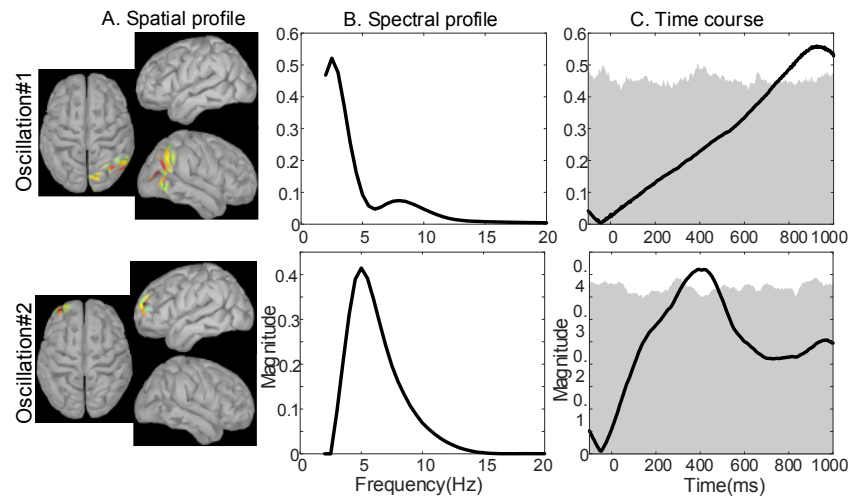

Fig. 3. Results of experiment data. A: spatial maps of extracted components. B: Spectral factors of the components. $\mathrm{C}$ : Time courses of components, averaged across trials in all subjects (black line). The grey shaded region represents the null distribution $\left(p_{\text {corrected }}<.05\right)$ based on a hypothesis that the response is not time locked to the tasks. Row 1 shows the Delta oscillation involved in comprehension of irony in right temporal-occipital junction after $800 \mathrm{~ms}$ of the stimulus onset. Row 2 demonstrates the Theta rhythm was elicited in Broca's area after $400 \mathrm{~ms}$ of the ironic stimulus onset.

As we all known, it is very complex to decode brain response to external stimulus. During a cognitive process, different brain rhythm would be emerging in different regions at different time, which causes the complexity of analysis for the EEG data. Therefore, the analysis for the data must account for temporal non-stationarity, spatial inhomogeneities, and spectral structure [20]. The time frequency representation of source level data based on wavelet transformation can well describe the property of the brain data. Tensor decomposition technique provides a means to extract information from such big and complex data.

\section{ACKNOWLEDGEMENT}

This work was partially supported by the Fundamental Research Funds for the Central Universities [DUT16JJ(G)03] in Dalian University of Technology in China, and National Natural Science Foundation of China (Grant No. 81471742) and the scholarships from China Scholarship Council (No. 201600090042). 


\section{REFERENCES}

[1] J. Damoiseaux et al., "Consistent resting-state networks across healthy subjects," Proceedings of the national academy of sciences, vol. 103, no. 37, pp. 13848-13853, 2006.

[2] S. M. Smith et al., "Correspondence of the brain's functional architecture during activation and rest," Proceedings of the National Academy of Sciences, vol. 106, no. 31, pp. 13040-13045, 2009.

[3] E. rol Başar, M. artin Schürmann, T. amer Demiralp, C. anan Başar-Eroglu, and A. hmet Ademoglu, "Event-related oscillations are 'real brain responses'-wavelet analysis and new strategies," International Journal of Psychophysiology, vol. 39, no. 2-3, pp. 91127, 2001.

[4] E. Başar, C. Başar-Eroğlu, S. Karakaş, and M. Schürmann, "Are cognitive processes manifested in event-related gamma, alpha, theta and delta oscillations in the EEG?," Neuroscience letters, vol. 259, no. 3, pp. 165-168, 1999.

[5] F. Cong et al., "Benefits of multi-domain feature of mismatch negativity extracted by non-negative tensor factorization from EEG collected by low-density array," International journal of neural systems, vol. 22, no. 06, p. 1250025, 2012.

[6] F. Cong et al., "Multi-domain feature extraction for small eventrelated potentials through nonnegative multi-way array decomposition from low dense array EEG," International journal of neural systems, vol. 23, no. 02, p. 1350006, 2013.

[7] D. Wang, Y. Zhu, T. Ristaniemi, and F. Cong, "Extracting multimode ERP features using fifth-order nonnegative tensor decomposition," Journal of neuroscience methods, vol. 308, pp. 240-247, 2018.

[8] F. Cong, Q.-H. Lin, L.-D. Kuang, X.-F. Gong, P. Astikainen, and T. Ristaniemi, "Tensor decomposition of EEG signals: a brief review," Journal of neuroscience methods, vol. 248, pp. 59-69, 2015.

[9] Y. Jonmohamadi, G. Poudel, C. Innes, and R. Jones, "Sourcespace ICA for EEG source separation, localization, and time-course reconstruction," NeuroImage, vol. 101, pp. 720-737, 2014.

[10] S. Sockeel, D. Schwartz, M. Pélégrini-Issac, and H. Benali, "Large-scale functional networks identified from resting-state EEG using spatial ICA," PloS one, vol. 11, no. 1, p. e0146845, 2016.

[11] F. Tadel, S. Baillet, J. C. Mosher, D. Pantazis, and R. M. Leahy, "Brainstorm: a user-friendly application for MEG/EEG analysis," Computational intelligence and neuroscience, vol. 2011, p. 8, 2011. [12] A. Delorme and S. Makeig, "EEGLAB: an open source toolbox for analysis of single-trial EEG dynamics including independent component analysis," Journal of neuroscience methods, vol. 134, no. 1, pp. 9-21, 2004.

[13] N. D. Sidiropoulos, L. De Lathauwer, X. Fu, K. Huang, E. E. Papalexakis, and C. Faloutsos, "Tensor decomposition for signal processing and machine learning," IEEE Transactions on Signal Processing, vol. 65, no. 13, pp. 3551-3582, 2017.

[14] A. Cichocki and A.-H. Phan, "Fast local algorithms for large scale nonnegative matrix and tensor factorizations," IEICE transactions on fundamentals of electronics, communications and computer sciences, vol. 92, no. 3, pp. 708-721, 2009.

[15] R. Bro and H. A. Kiers, "A new efficient method for determining the number of components in PARAFAC models," Journal of Chemometrics: A Journal of the Chemometrics Society, vol. 17, no. 5, pp. 274-286, 2003.

[16] D. Kim, K. Kay, G. L. Shulman, and M. Corbetta, "A new modular brain organization of the BOLD signal during natural vision," Cerebral Cortex, pp. 1-17, 2017.
[17] G. C. O'neill et al., "Measurement of dynamic task related functional networks using MEG," NeuroImage, vol. 146, pp. 667678, 2017.

[18] D. Mobbs, M. D. Greicius, E. Abdel-Azim, V. Menon, and A. L. Reiss, "Humor modulates the mesolimbic reward centers," Neuron, vol. 40, no. 5, pp. 1041-1048, 2003.

[19] M. Shibata et al., "Time course and localization of brain activity in humor comprehension: An ERP/sLORETA study," Brain research, vol. 1657, pp. 215-222, 2017.

[20] M. J. Brookes et al., "Measuring temporal, spectral and spatial changes in electrophysiological brain network connectivity," Neuroimage, vol. 91, pp. 282-299, 2014. 